[9] G. Tenenbaum, Estimations asymptotiques de fonetions arithmétiques liées aux diviseurs, Thèse, Bordeaux, 30 arril 1976.

[10] - Sur deux fonctions de diviseurs, J. London Math. Soc. (2) 14 (1967), p. 521-526.

[11] - Sur la probabilité qu'un entier possède un diviseur dans un intervalle donné, preprint.

IABORATOIRE DE THEOORIE DES NOMBRES Marseille, France

\section{Special values of the dilogarithm function}

by

J. H. LoxтoN (Kensington, New South Tales, Australia)

1. Introduction. The dilogarithm funetion defined, for suitable $z$, by

$$
\mathrm{Li}_{2}(z)=\sum_{n=1}^{\infty} z^{n} / n^{2}=-\int_{0}^{\tilde{z}} \frac{\log (1-t)}{t} d t,
$$

is one of the lesser transcendental functions. Nonetheless, as Lewin's treatise [3] demonstrates, it has a very respectable pedigree and a wealth of curious properties.

The present work will be concerned with some nnexpected relations between the values of the dilogarithm function at certain algebraic integers. Lewin [4] has shown how interesting relations can be obtained by specializing Abel's functional equation for the dilogarithm function, but this technique does not seem to yield all the known results. Richmond and Szekeres [ 5$]$ have introduced a different idea. They apply the oircle method to obtain asymptotic formulae for the power series coefficients of the functions occurring on the two sides of a partition identity of Andrews and Gordon. A comparison of the two formulae then yields non-trivial numerical relations for the dilogarithm function. I shall exploit the same principle here. The investigation has produced some new partition identities of the same type as the celebrated Rogers-Ramanujan identities and some new relations between values of the dilogarithm function. In particular, I shall prove a formula conjectured by Lewin [4] which had apparently resisted more direct attacks. Despite this success, these ideas do not seem to touch the central problem here which is to explain the mechanism leading to such a profusion of identities.

2. The dilogarithm relations. It is most convenient to work with the function

$$
L(z)=\mathrm{Li}_{2}(z)+\frac{1}{2} \log z \cdot \log (1-z)
$$

instead of with the dilogarithm function itself. To avoid problems with complex logarithms, the argument $\approx$ will be restricted to the interval 
$0<z<1$. The function $L(z)$ satisfies the functional equations

$$
L(z)+L(\mathfrak{X}-z)=\pi^{2} / 6
$$

and

$$
L(z)=L(z /(1+z))+\frac{1}{2} L\left(z^{2}\right),
$$

both of which can be readily verified by differentiation.

The functional equations for $L(z)$ yield evaluations of the dilogarithm function at some special algebraic points, namely Euler's results

$$
\begin{array}{ll}
\text { (Li1) } & L(1)=\pi^{2} / 6 \\
\text { and } & L\left(\frac{1}{2}\right)=\pi^{2} / 12, \\
\text { (L2) } &
\end{array}
$$

and Landen's results

$$
L\left(\frac{1}{2}(\sqrt{5}-1)\right)=\pi^{2} / 10 \quad \text { and } \quad L\left(\frac{1}{2}(3-\sqrt{5})\right)=\pi^{2} / 15 .
$$

Apparentily, there are no other algebraic points at which there is such an elementary evaluation of the dilogarithm function. However, there are many identities relating the values of the dilogarithm funotion at various powers of algebraic numbers. A simple example is

$$
6 L(1 / 3)-L(1 / 9)=\pi^{2} / 3,
$$

which is easily obtained from the functional equations for $L(z)$. Again, if $a=\sqrt{2}-1$, we have the relations

$$
4 L(\alpha)-L\left(a^{2}\right)=\pi^{2} / 4
$$

and

$$
4 L(\alpha)+4 L\left(\alpha^{2}\right)-L\left(\alpha^{4}\right)=5 \pi^{2} / 12,
$$

both obtained by Lewin [4]. A similar relation not given by Lewin is

$$
12 L(\beta)+3 L\left(\beta^{2}\right)-2 L\left(\beta^{3}\right)=5 \pi^{2} / 6, \quad \beta=\frac{1}{2}(\sqrt{3}-1) .
$$

Watson [8] found three relations involving the roots of the cubic $x^{3}+$ $+2 x^{2}-x-1$, as follows. If the roots of this cubic are denoted by $\gamma,-\delta$ and $-1 / \varepsilon$, so that $\gamma, \delta$ and $\varepsilon$ all lie between 0 and 1 , then

and

$$
\begin{aligned}
L(\gamma)-L\left(\gamma^{2}\right) & =\pi^{2} / 42, & & \gamma=1 /(2 \cos 2 \pi / 7), \\
2 L(\delta)+L\left(\delta^{2}\right) & =5 \pi^{2} / 21, & \delta & =1 /(2 \cos \pi / 7),
\end{aligned}
$$

$$
2 L(\varepsilon)+L\left(\varepsilon^{2}\right)=4 \pi^{2} / 21, \quad \varepsilon=2 \cos 3 \pi / 7 .
$$

Watson obtained these formulae by repeated use of the basic functional equations given above. Slightly more complicated relations attach to the roots of the cubic $x^{3}+3 x^{2}-1$. If the roots of this cubic are denoted by $\zeta,-\eta$ and $-1 / \theta$, so that $\zeta, \eta$ and $\theta$ again lie between 0 and 1 , then

(Li12) $\quad 6 L(\eta)-9 L\left(\eta^{2}\right)-2 L\left(\eta^{3}\right)+L\left(\eta^{6}\right)=-\pi^{2} / 9, \quad \eta=1(2 \cos 2 \pi / 9)$

and

(L13) $\quad 6 L(\theta)-9 L\left(\theta^{2}\right)-2 L\left(\theta^{3}\right) \div L\left(\theta^{6}\right)=\pi^{2} / 9, \quad \theta=2 \cos t \pi / 9$.

Here, (L11) was discovered by the asymptotic analysis described below. Lewin observed the parallel with Watson's three identities and conjectured the relations (L12) and (L13) in [1]. (The parallel is perhaps clearer in the alternative notation

$$
\begin{aligned}
3 \mathrm{Li}_{2}(\zeta)+3 \mathrm{Li}_{2}\left(\zeta^{2}\right)-\mathrm{Li}_{2}\left(\zeta^{3}\right) & =\pi \pi^{2} / 18-3 \log ^{2} \zeta \\
3 \mathrm{Li}_{2}(-\eta)+3 \mathrm{Li}_{2}\left(\eta^{2}\right)-\mathrm{Li}_{2}\left(-\eta^{3}\right) & =\pi^{2} / 18-3 \log ^{2} \eta \\
3 \mathrm{Li}_{2}(-\theta)+3 \mathrm{Li}_{2}\left(\theta^{2}\right)-\mathrm{Li}_{2}\left(-\theta^{3}\right) & \left.=-\pi^{2} / 18 .\right)
\end{aligned}
$$

The method to be described below yields the relations (L11) and (L12), but I have not been able to obtain (L13). As far as I know, none of these three relations has a more direct proof.

3. The partition identities. The relations in the previous section will be derived by applying the circle method to certain identities which express basic hypergeometric series as infinite products. Since the analysis is rather rough, many of these identities typieally yield the same dilogarithm relation and the list below is only intended to be a representative selection. Many of the identities appear in Slater's list [6] of 130 identities of this type. These will be referred to as (S1) to (S130) in what follows. In stating the identities, I use the standard abbreviations

$$
(a)_{n}=(a ; q)_{n}=(1-a)(1-a q) \ldots\left(1-a q^{n-1}\right),
$$

for $n$ a positive integer,

$$
(a)_{\infty}=(a ; q)_{\infty}=\lim _{n \rightarrow \infty}(a ; q)_{n}
$$

and, in general, for any real number $n$,

$$
(a)_{n}=(a ; q)_{n}=(a ; q)_{\infty} /\left(a q^{n} ; q\right)_{\infty} .
$$

The firs few identities are well known, namely Euler's identities

and

$$
\sum_{n=0}^{\infty} q^{n} /(q)_{n}=1 /(q)_{\infty}
$$

$(\mathrm{P} 2=\mathrm{S} 2)$

$$
\sum_{n=0}^{\infty} q^{n(n+1) / 2} /(q)_{n}=1 /\left(q ; q^{2}\right)_{\infty}
$$


and the first Rogers-Ramanujan identity

$(\mathrm{P} 3=\mathrm{S} 18)$

$$
\sum_{n=0}^{\infty} q^{n^{2}} /(q)_{n}=1 /\left(q ; q^{5}\right)_{\infty}\left(q^{4} ; q^{5}\right)_{\infty}
$$

The following identities are variations on the same theme, becoming steadily more complex.

$$
\begin{aligned}
& (\mathrm{P} 4=\mathrm{S} 47) \quad \sum_{n=0}^{\infty}\left(-1 ; q^{2}\right)_{n} q^{n^{2}} /(q)_{2 n}=\left(-q ; q^{2}\right)_{\infty} /\left(q ; q^{2}\right)_{\infty}, \\
& (\mathrm{P} 5=\mathrm{S} 8) \quad \sum_{n=0}^{\infty}(-q)_{n} q^{\frac{1}{n} n(n+1)} /(q)_{n}=(-q)_{\infty} /\left(q^{2} ; q^{4}\right)_{\infty}, \\
& (\mathrm{P} 6=\mathrm{S} 37) \quad \sum_{n=0}^{\infty}(-q)_{n}\left(-q ; q^{2}\right)_{n} q^{\frac{1 n}{n(n+1)}} /(q)_{2 n+1} \\
& =(-q)_{\infty}\left(q^{3} ; q^{8}\right)_{\infty}\left(q^{5} ; q^{8}\right)_{\infty}\left(q^{8} ; q^{8}\right)_{\infty} /(q)_{\infty} \\
& (\mathrm{P} 7=\mathrm{S} 70) \quad \sum_{n=0}^{\infty}(-q)_{n+1}\left(q^{3} ; q^{3}\right)_{n} q^{\frac{1}{n n(n+3)} /(q)_{n}(q)_{2 n+2}} \\
& =(-q)_{\infty}\left(q^{3} ; q^{18}\right)_{\infty}\left(q^{15} ; q^{18}\right)_{\infty}\left(q^{18} ; q^{18}\right)_{\infty} /(q)_{\infty}, \\
& (\mathrm{P} 8=\mathrm{S} 33) \quad \sum_{n=0}^{\infty} q^{2 n^{2}} /\left(q^{2} ; q^{2}\right)_{n}(-q)_{2 n} \\
& =\left(q^{3} ; q^{7}\right)_{\infty}\left(q^{4} ; q^{7}\right)_{\infty}\left(q^{7} ; q^{7}\right)_{\infty} /\left(q^{2} ; q^{2}\right)_{\infty}, \\
& (\mathrm{P} 9=\mathrm{S} 61) \quad \sum_{n=0}^{\infty} q^{n^{2}} /(q)_{n}\left(q ; q^{2}\right)_{n}=\left(q^{6} ; q^{14}\right)_{\infty}\left(q^{8} ; q^{14}\right)_{\infty}\left(q^{14} ; q^{14}\right)_{\infty} /(q)_{\infty} \text {; } \\
& (\mathrm{P} 10=\mathrm{S} 82) \quad \sum_{n=0}^{\infty}(-q)_{n} q^{q n(n+3)} /(q)_{2 n+1} \\
& =(-q)_{\infty}\left(q^{3} ; q^{7}\right)_{\infty}\left(q^{4} ; q^{7}\right)_{\infty}\left(q^{7} ; q^{7}\right)_{\infty}\left(q ; q^{14}\right)_{\infty}\left(q^{13} ; q^{14}\right)_{\infty} /(q)_{\infty} \\
& (\mathrm{P} 11=\mathrm{S} 92) \quad \sum_{n=0}^{\infty}\left(q^{3} ; q^{3}\right)_{n} q^{n(n+1)} /(q)_{n}(q)_{2 n+1} \\
& =\left(q^{9} ; q^{27}\right)_{\infty}\left(q^{38} ; q^{27}\right)_{\infty}\left(q^{27} ; q^{27}\right)_{\infty} /(q)_{\infty}
\end{aligned}
$$

The remaining identities do not appear to be in Slater's list.

$$
\begin{aligned}
& 1+\sum_{n=1}^{\infty}\left(-q^{3} ; q^{3}\right)_{n-1} q^{n(n+1)} /(-q)_{n-1}(q)_{2 n} \\
& \quad=\left(q ; q^{3}\right)_{\infty}\left(q^{8} ; q^{9}\right)_{\infty}\left(q^{9} ; q^{9}\right)_{\infty}\left(q^{7} ; q^{18}\right)_{\infty}\left(q^{11} ; q^{18}\right)_{\infty} /(q)_{\infty},
\end{aligned}
$$

$(\mathrm{P} 12$ bis $) \quad 1+\sum_{n=1}^{\infty}\left(-q^{3} ; q^{3}\right)_{n-1} q^{n^{2}} /(-q)_{n-1}(q)_{2 n}$

$$
=\left(q^{2} ; q^{9}\right)_{\infty}\left(q^{7} ; q^{9}\right)_{\infty}\left(q^{9} ; q^{9}\right)_{\infty}\left(q^{5} ; q^{18}\right)_{\infty}\left(q^{13} ; q^{18}\right)_{\infty} /(q)_{\infty}
$$

$$
1-\sum_{n=1}^{\infty}\left(-q^{3} ; q^{3}\right)_{n-2} q^{n-1}\left(1-q^{n-1}-q^{n}-q^{n+1}+q^{3 n}\right) /(-q)_{n-2}(q)_{n n}
$$$$
=\left(q ; q^{6}\right)_{\infty}\left(q^{5} ; q^{6}\right)_{\infty} /(q)_{\infty} \text {. }
$$

The final identity is the nearest that $I$ have been able to come to finding an identity for Lewin's conjectured dilogarithm relation (L13). It seems worth including here to show the limitations of the present methods:

In the quest for the lost identity to match the relation (L13), I worked through all 130 identities in Slater's list. [6]. The theorem in Section 5 below cannot be applied in every case, but when it does apply, it gives a useful independent check on the identity. The asymptotic analysis located two identities which should be corrected as follows:

$$
\begin{aligned}
& \sum_{n=0}^{\infty}(-1)_{n} q^{n^{2}} /(q)_{n}\left(q ; q^{2}\right)_{n}=\left(-q ; q^{3}\right)_{\infty}\left(-q^{2} ; q^{3}\right)_{\infty}\left(q^{3} ; q^{3}\right)_{\infty} /(q)_{\infty} \\
& \begin{array}{c}
\sum_{n=0}^{\infty}(-1)_{2 n} q^{n^{2}} /\left(q^{2} ; q^{2}\right)_{n}\left(q^{2} ; q^{4}\right)_{n} \\
\quad=\left(-q ; q^{2}\right)_{\infty}\left(-q^{2} ; q^{2}\right)_{\infty}\left(-q ; q^{4}\right)_{\infty}\left(-q^{3} ; q^{4}\right)_{\infty}
\end{array}
\end{aligned}
$$

4. Proofs of the partition identities. I will sketch the derivations of the identities (P12) and (P13), following, more or less, the method developed by Slater $([6], p .151)$. This rests on the following very general series transformation first exploited by Bailey. If

$$
\beta_{n}=\sum_{r=0}^{n} \alpha_{r} u_{n-r} v_{n+r} \quad \text { and } \quad \gamma_{n}=\sum_{r=n}^{\infty} \delta_{r} u_{r-n} v_{r+n},
$$

then, subject to proper conditions for convergence,

$$
\sum_{n=0}^{\infty} \alpha_{n} \gamma_{n}=\sum_{n=0}^{\infty} \beta_{n} \delta_{n}
$$

Proof of (P12). Following Slater, let

$$
u_{n}=1 /(q)_{n}, \quad v_{n}=1 /(x)_{n}, \quad \delta_{n}=(y)_{n}(z)_{n} x^{n} / y^{n} z^{n} .
$$

The series for $\gamma_{n}$ can be summed by the basic analogue of Ganss's theorem ([1], Corollary 2.4), giving

$$
\gamma_{n}=\frac{(x / y)_{\infty}(x / z)_{\infty}}{(x)_{\infty}(x / y z)_{\infty}} \frac{(y)_{n}(z)_{n} x^{n}}{(x / y)_{n}(x / z)_{n} y^{n} z^{n}} .
$$


Again, following Slater, $\alpha_{n}$ and $\beta_{n}$ are determined by specializing Bailey's summation

(2) $\quad \sum_{n=-\infty}^{\infty} \frac{\left(1-a q^{2 n}\right)(b)_{n}(c)_{n}(d)_{n}(e)_{n} a^{2 n} q^{n}}{(1-a)(a q / b)_{n}(a q / c)_{n}(a q / d)_{n}(a q / e)_{n} b^{n} c^{n} d^{n} e^{n}}$

$=\frac{(q)_{\infty}(q / a)_{\infty}(a q)_{\infty}(a q / b c)_{\infty}(a q / b d)_{\infty}(a q / b e)_{\infty}(a q / e d)_{\infty}(a q / c e)_{\infty}(a q / d e)_{\infty}}{(q / b)_{\infty}(q / e)_{\infty}(q / d)_{\infty}(q / e)_{\infty}(a q / b)_{\infty}(a q / e)_{\infty}(a q / d)_{\infty}(a q / e)_{\infty}\left(a^{2} q / b c d e\right)_{\infty}}$

for a certain well-poised bilateral hypergeometric series. If $a=q^{1 / 3}$, $b=q^{-n / 3}, c=q^{(1-n) / 3}, d=q^{(2-n) / 3}$ and $e=-q^{2 / 3}$, and then $q$ is replaced by $q^{3}$, this yields

$$
\sum_{r=-[n / 3]}^{[n / 3]} \frac{\left(1-q^{6 r+1}\right) q^{\frac{3}{\left(9 r^{2}-3 r\right]}}}{(q)_{n+3 r+1}(q)_{n-3 r}}=\frac{\left(-q^{3} ; q^{3}\right)_{n-1}}{(q)_{2 n}(-q)_{n-1}} .
$$

Since

$$
\left(1-q^{6 r+1}\right) q^{\frac{1}{\left(3\left(9 r^{2}-3 r\right)\right.}}=q^{\frac{1}{\left(2\left(9 r^{2}+3 r\right)-n\right.}}\left\{\left(1-q^{n+3 r+1}\right)-\left(1-q^{n-3 r}\right)\right\},
$$

the left-hand side of (3) can be re-Fritten as

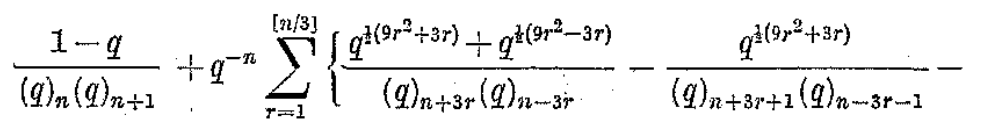

$$
\begin{aligned}
& \left.-\frac{q^{\frac{1}{\left(2\left(9 r^{2}-3 r\right)\right.}}}{(q)_{n-3 r+1}(q)_{n+3 r-1}}\right\}
\end{aligned}
$$

Thus the prescriptions above are satisfied by taking

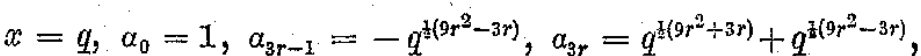

$$
\begin{aligned}
& \alpha_{3 r+1}=-q^{i\left(9 r^{2}+3 r\right)}
\end{aligned}
$$

and

$$
\beta_{n}=\left(-q^{3} ; q^{3}\right)_{n-1} q^{n} /(q)_{2 n}(-q)_{n-1} .
$$

Using the above values for $\alpha_{n}, \beta_{n}, \gamma_{n}$ and $\delta_{n}$ in (1), and letting $y$ and $z$ tend to infinity, leads to

$$
\begin{aligned}
& 1+\sum_{n=1}^{\infty}\left(-q^{3} ; q^{3}\right)_{n-1} q^{n^{2}+n} /(q)_{2 n}(-q)_{n-1}
\end{aligned}
$$

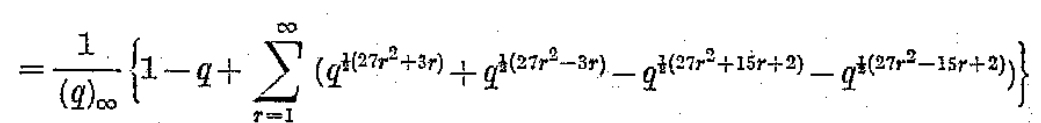

$$
\begin{aligned}
& =\left\{\left(-q^{12} ; q^{27}\right)_{\infty}\left(-q^{15} ; q^{27}\right)_{\infty}\left(q^{27} ; q^{27}\right)_{\infty}-q\left(-q^{6} ; q^{27}\right)_{\infty}\left(-q^{21} ; q^{27}\right)_{\infty} \times\right. \\
& \left.\times\left(q^{27} ; q^{27}\right)_{\infty}\right\} /(q)_{\infty}
\end{aligned}
$$

by Jacobi's triple product identity ([1], Theorem 2.8). Finally, the right side can be expressed as a single product by means of Watson's quintuple product identity ([2], (2.1)), giving (P12). Similarly, writing

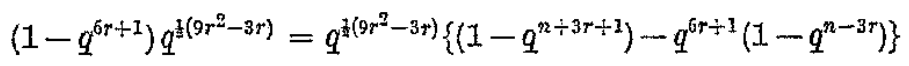

on the left side of (3) leads to the transformation

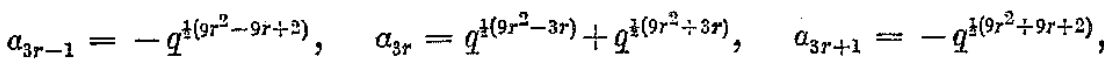

$$
\begin{aligned}
& \beta_{n}=\left(-q^{3} ; q^{3}\right)_{n-1} /(q)_{2 n}(-q)_{n-1},
\end{aligned}
$$

with $\alpha_{0}=\beta_{0}=1$ and $x=q$, and then letting $y$ and $z$ tend to infinity gives (P12 bis).

Proof of (P13). For this case, let

$$
\begin{aligned}
& u_{n}=1 /\left(q^{-1} ; q^{-1}\right)_{n}=(-1)^{n} q^{\frac{3}{3} n(n+1)} /(q)_{n}, \\
& v_{n}=1 /\left(x^{-1} ; q^{-1}\right)_{n}=(-1)^{n} x^{n} q^{\frac{1}{n(n-1)}} /(x)_{n}
\end{aligned}
$$

and

$$
\begin{aligned}
\delta_{n} & =\left(y^{-1} ; q^{-1}\right)_{n}\left(z^{-1} ; q^{-1}\right)_{n} y^{n} z^{n}(1-q / w) / x^{n} w^{n} \\
& =(y\rangle_{n}(z)_{n}(1-q / w) / x^{n} w^{n} q^{n^{2}-n} .
\end{aligned}
$$

The series for $\gamma_{n}$ can be summed in the limit when $w$ tends to $q$, as follows. With the above definitions,

$$
\begin{aligned}
\gamma_{n} & =\sum_{r=n}^{\infty} \frac{(y)_{r}(z)_{r}}{(q)_{r-n}(x)_{r+n}}\left(\frac{q}{w}\right)^{r}\left(1-\frac{q}{w}\right) x^{n} q^{n^{2}-n} \\
& =\sum_{s=0}^{\infty} \frac{\left(q^{n} y\right)_{s}\left(q^{n} z\right)_{s}}{(q)_{s}\left(q^{2 n} x\right)_{s}}\left(\frac{q}{w}\right)^{s}\left(1-\frac{q}{w}\right) \frac{(y)_{n}(z)_{n} x^{n} q^{n^{2}}}{(x)_{2 n} w^{n}}
\end{aligned}
$$

By the third iterate of Heine's transformation ([1], (3.3.13)), the above is

$$
\sum_{s=0}^{\infty} \frac{\left(q^{n} x / y\right)_{s}\left(q^{n} x / z\right)_{s}}{(q)_{s}\left(q^{2 n} x\right)_{s}}\left(\frac{q y z}{w x}\right)^{s} \frac{(q y z / w x)_{\infty}(y)_{n}(z)_{n} x^{n} q^{n^{2}}}{\left(q^{2} / w\right)_{\infty}(x)_{2 n} w^{n}}
$$

Now, it is possible to set $w=q$, whenee, by the basic analogue of Gauss's theorem,

$$
\gamma_{n}=x^{n} q^{n^{2}-n}(y)_{\infty}(z)_{\infty} /(q)_{\infty}(x)_{\infty}
$$

The sequences $\alpha_{n}$ and $\beta_{n}$ can be determined by replacing $q$ by $q^{-1}$ in the derivation of (P12). Thus we talke $x=q$,

$\left.a_{3 r-1}=-q^{-1\left(9 r^{2}-3 r\right)}\right), \quad a_{3 r}=q^{-\left(9 r^{2}+3 r\right) / 2}+q^{-\left(9 r^{2}-3 r\right) / 2}, \quad a_{3 r+1}=-q^{-1\left(9 r^{2}+3 r\right)}$ 
and

$$
\begin{aligned}
\beta_{n}=\left(-q^{-3} ; q^{-3}\right)_{n-1} q^{-n} /\left(q^{-1} ; q^{-1}\right)_{2_{n}}\left(-q^{-1} ; q^{-1}\right)_{n-1} \\
\quad=\left(-q^{2} ; q^{3}\right)_{n-1} q^{n^{2}+n} /(q)_{2_{n}}(-q)_{n-1},
\end{aligned}
$$

with the convention that $a_{0}=\beta_{0}=1$. Now

$$
\begin{aligned}
& \sum_{n=0}^{\infty} \beta_{n} \delta_{n}=\sum_{n=0}^{\infty} \frac{\left(-q^{3} ; q^{3}\right)_{n-1}(y)_{n}(z)_{n}}{(q)_{2 n}(-q)_{n-1}}\left(\frac{q}{w}\right)^{n}\left(1-\frac{q}{w}\right) \\
& =1-\sum_{n=1}^{\infty}\left\{\frac{\left(-q^{3} ; q^{3}\right)_{n-2}(y)_{n-1}(z)_{n-1}}{(q)_{2 n-2}(-q)_{n-2}}-\frac{\left(-q^{3} ; q^{3}\right)_{n-1}(y)_{n}(z)_{n}}{(q)_{2 n}(-q)_{n-1}}\right\}\left(\frac{q}{w}\right)^{n} .
\end{aligned}
$$

If $w=q$ and $y$ and $z$ tend to zero, this gives

$$
\sum_{n=0}^{\infty} \beta_{n} \delta_{n}=1-\sum_{n=1}^{\infty} \frac{\left(-q^{3} ; q^{3}\right)_{n-2}}{(q)_{2 n}(-q)_{n-2}} q^{n-1}\left(1-q^{n-1}-q^{n}-q^{n+1}+q^{3 n}\right) .
$$

On the other hand, again letting $y$ and $z$ tend to zero,

$$
\sum_{n=0}^{\infty} \alpha_{n} \gamma_{n}=\sum_{n=0}^{\infty} a_{n} q^{n^{2}} /(q)_{\infty}^{2}
$$

and this can be summed by the method used in the derivation of (P12) to give

$$
\sum_{n=0}^{\infty} a_{n} \gamma_{n}=\left(q ; q^{6}\right)_{\infty}\left(q^{5} ; q^{6}\right)_{\infty} /(q)_{\infty} .
$$

Thus, (P13) follows from (1).

5. Asymptotic analysis of the series. The estimation of the power series coefficients of the series appearing in the partition identities of Section 3 will be accomplished by means of the following theorem.

THEOREM. Consider the power series

$$
\sum_{n=0}^{\infty} q^{n(a n+b) / 2} / \prod_{j=1}^{r}\left(q^{c_{j}} ; q^{d_{j}}\right)_{n}^{\varepsilon_{j}}=\sum_{k=0}^{\infty} a_{k} q^{k_{k}}
$$

where $a, b, c_{j}, d_{j}$ and $\varepsilon_{j}$ are integers satisfying $a \geqslant 0, b>0$ if $a=0$, $a \equiv b(\bmod 2)$ and $d_{j}>0$. Suppose that the power series expansion of each of the products $\prod_{j=1}^{r}\left(q^{c_{j}} ; q^{d_{j}}\right)_{n}^{-\varepsilon_{j}}$ has positive coefficients, and that the equation $\prod_{j=1}^{r}\left(1-x^{a_{j}}\right)^{\varepsilon_{j}}=x^{c^{a}} \cdot h_{\text {has }}$ a unique root, $\mu$ say, between 0 and 1 . Then

$$
\left(\log a_{k}\right)^{2} / 4 k \rightarrow \sum_{j=1}^{r}\left(\varepsilon_{j} / d_{j}\right) L\left(1-\mu^{a_{j}}\right), \quad \text { as } k \rightarrow \infty .
$$

Proof. The argument follows the method developed by Szekeres 17]. Consider first the power series

$$
q^{n(a n+b) / 2} / \prod_{j=1}^{r}\left(q^{c_{j}} ; q^{a_{j}}\right)_{n}^{\varepsilon_{j}}=\sum_{k=0}^{\infty} a_{k n} q^{k}
$$

The $a_{k n n}$ can be obtained by Cauchy's formula, applied to a suitable circle described by $q=e^{-\beta+i \theta}(-\pi \leqslant \theta \leqslant \pi)$ with radius $e^{-\beta}$ less than 1 . Thus

$$
\begin{aligned}
a_{k n}=\frac{1}{2 \pi} \int_{-\pi}^{\pi} \exp \left\{-\sum_{j=1}^{r} \sum_{j=0}^{n-1} \varepsilon_{j} \log \left(1-e^{-(\beta-i \theta)\left(\pi_{j} p+c_{j}\right)}\right)+\right. \\
\left.\quad+\left(k-\frac{1}{2} n(a n+b)\right)(\beta-i \theta)\right\} d \theta .
\end{aligned}
$$

The radius, $e^{-\beta}$, of the circle of integration is determined by the saddlepoint condition

$$
\sum_{j=1}^{r} \sum_{\nu=0}^{n-1} \varepsilon_{j}\left(d_{j} \nu+c_{j}\right) /\left(e^{\left(a_{j} p+c_{j}\right) \beta}-1\right)=k-\frac{1}{2} n(a n+b) .
$$

With this choice of $\beta$, the method of [7], Section 2, gives

(5) $\quad \log a_{k n}=\left(k-\frac{1}{2} n(a n+b)\right) \beta-\sum_{j=1}^{r} \sum_{p=0}^{n-1} \varepsilon_{j} \log \left(1-e^{-\left(a_{j^{*}}+c_{j}\right) \beta}\right)+$

$$
+\frac{1}{2} \log \left(\beta^{3} / 2 \pi A\right)+0(1)
$$

where

$$
A=\sum_{j=1}^{r} \frac{\varepsilon_{j}}{d_{j}} \int_{0}^{a_{j} u} \frac{t^{2} e^{t}}{\left(e^{t}-1\right)^{2}} d t \quad \text { and } \quad u=\beta n .
$$

By the Enler-Maclaurin sum formula, applied to (4),

$$
\frac{1}{\beta^{2}} \sum_{j=1}^{r} \frac{\varepsilon_{j}}{d_{j}} \int_{0}^{d_{j} u} \frac{t}{e^{t}-1} d t=k-\frac{a u^{2}}{2 \beta^{2}}+O\left(k^{1 / 2}\right),
$$

that is

$$
k=\frac{1}{\beta^{2}}\left\{\sum_{j=1}^{r} \frac{\varepsilon_{j}}{d_{j} \int_{0} u} \frac{t}{e^{t}-1} d t+\frac{1}{2} a u^{2}\right\}+O\left(k^{1 / 2}\right),
$$

so that $\beta$ is of order $k^{-1 / 2}$. Again, applying the Euler-Maclarurin sum formula to (5) in conjunction with (4),

(7) $\quad \log a_{k n}=\frac{1}{\beta} \sum_{j=1}^{r}\left\{\frac{2 \varepsilon_{j}}{d_{j}} \int_{0}^{a_{j}{ }^{*}} \frac{t}{e^{t}-1} d t-\varepsilon_{j} u \log \left(1-e^{-a_{j} u}\right)\right\}+o\left(k^{1 / 2}\right)$. 
The next step is to determine $n$ sc that this expression for $\log a_{k n}$ is maximal. Let the difference operator $\Delta$ denote differences occasioned by moving from $n$ to $n+1$. From (4)

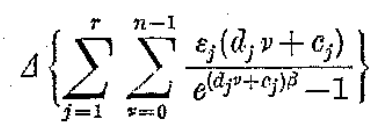

$$
\begin{aligned}
& =-\sum_{j=1}^{r} \sum_{v=0}^{n-1} \frac{\varepsilon_{j}\left(d_{j} p+c_{j}\right)^{2} e^{\left(a_{j} y+c_{j}\right) \beta}}{\left(e^{\left(a_{j} p+c_{j} j\right)^{\beta}}-1\right)^{2}} \Delta \beta+\sum_{j=1}^{r} \frac{\varepsilon_{j}\left(d_{j} n+c_{j}\right)}{e^{\left(a_{j} n+c_{j}\right) \beta}-1}+o(1) \\
& =-a n-\frac{1}{2} b
\end{aligned}
$$

and, as in [7], Section 4, this leads to

$$
\Delta \beta=\beta^{3}\left\{\sum_{j=1}^{r} \frac{\varepsilon_{j}\left(d_{j} n+c_{j}\right)}{e^{\left(d_{j} n+c_{j}\right)^{\beta}}-1}+a n\right\} /\left\{\sum_{j=1}^{r} \frac{\varepsilon_{j}}{d_{j}} \int_{0}^{d_{j} u} \frac{t^{2} e^{t}}{\left(e^{t}-1\right)^{2}} d t\right\}+o\left(k^{-1}\right) .
$$

In the same way, (5) gives

$$
\Delta \log a_{k n}=-\sum_{j=1}^{r} \varepsilon_{j} \log \left(1-e^{-d_{j} u}\right)-a \imath+o(1) .
$$

The condition for $\log a_{k n}$ to be maximal is that this last expression should vanish, that is

$$
\sum_{j=1}^{r} \varepsilon_{j} \log \left(1-e^{-d_{j} u}\right)=-a u
$$

Since $0<e^{-u}<1$, the hypotheses of the theorem fix $e^{-u}=\mu$. Substituting (8) in (6) and (7) gives the maximum

$$
\log a_{k n}=2 k^{1 / 2}\left\{\sum_{j=1}^{r} \frac{\varepsilon_{j}}{d_{j}} \int_{0}^{d_{j} u} \frac{t}{e^{t}-1} d t-\frac{1}{2} \varepsilon_{j} u \log \left(1-e^{-d_{j} u}\right)\right\}^{1 / 2}+o\left(k^{1 / 2}\right)
$$

in which

$$
\int_{0}^{d_{j} u} \frac{t}{e^{t}-1} d t-\frac{1}{2} d_{j} u \log \left(1-e^{-d_{j} u}\right)=L\left(1-\mu^{d_{j}}\right) .
$$

Finally, $a_{k}=\sum a_{k n}$, where the sum over $n$ has at most $k$ terms, all of them positive, so that

$$
\log a_{k}=2 k^{1 / 2}\left\{\sum_{j=1}^{r} \frac{\varepsilon_{j}}{d_{j}} L\left(1-\mu^{d_{j}}\right)\right\}^{1 / 2}+o\left(k^{1 / 2}\right) .
$$

6. Proofs of the dilogarithm relations. To illustrate the application of the theorem, I shall sketch the derivation of the relation (L12). The identity (P12) can be re-written as

$$
\begin{aligned}
& 1+\frac{q^{2}}{(1-q)\left(1-q^{2}\right)} \sum_{n=0}^{\infty} \frac{(q)_{n}\left(q^{6} ; q^{6}\right)_{n} q^{n^{2}+3 n}}{\left(q^{2} ; q^{2}\right)_{n}\left(q^{3} ; q^{2}\right)_{n}\left(q^{4} ; q^{2}\right)_{n}\left(q^{3} ; q^{3}\right)_{n}} \\
& =\left(q ; q^{9}\right)_{\infty}\left(q^{8} ; q^{9}\right)_{\infty}\left(q^{9} ; q^{9}\right)_{\infty}\left(q^{7} ; q^{18}\right)_{\infty}\left(q^{11} ; q^{18}\right)_{\infty} /(q)_{\infty} \text {. }
\end{aligned}
$$

Let $\sum a_{k} q^{k}$ be the power series expansion of either side of this identity. The estimate for $\log a_{k}$ implied by the left side of the identity is the same as that obtaining for the series

$$
\sum_{n=0}^{\infty} \frac{(q)_{n}\left(q^{6} ; q^{6}\right)_{n} q^{n^{2}+3 n}}{\left(q^{2} ; q^{2}\right)_{n}\left(q^{3} ; q^{3}\right)_{n}\left(q^{4} ; q^{2}\right)_{n}\left(q^{3} ; q^{3}\right)_{n}} .
$$

As in the theorem, let $\mu$ be the real root between 0 and 1 of the equation

$$
\left(1-x^{2}\right)^{3}\left(1-x^{3}\right)=x^{2}(1-x)\left(1-x^{6}\right),
$$

that is, $x^{3}-3 x^{2}+1=0$, so that $\mu=1 /(2 \cos 2 \pi / 9)$. By the theorem,

$$
\left(\log a_{k}\right)^{2} \sim 4 k\left\{\frac{3}{2} L\left(1-\mu^{2}\right)+\frac{1}{3} L\left(1-\mu^{3}\right)-L(1-\mu)-\frac{1}{6} L\left(1-\mu^{6}\right)\right\},
$$

as $k \rightarrow \infty$. The estimate for $\log a_{k}$ from the right side of the identity follows readily from the well-known estimate for the partition function; namely, if

$$
\prod_{n=1}^{\infty}\left(1-q^{n}\right)^{-1}=\sum_{k=0}^{\infty} p(k i) q^{k}
$$

then

$$
\log p(k) \sim \pi(2 k / 3)^{1 / 2}
$$

as $k \rightarrow \infty$. The right side of the identity is obtained by omitting 8 residue classes modulo 18 from the last infinite product, whence

$$
\left(\log a_{k}\right)^{2} \sim \frac{10}{18}(\log p(k))^{2} \sim 10 \pi^{2} \% / 2 \pi,
$$

as $k \rightarrow \infty$. Now $(\mathrm{L} 12)$ follows on equating the two evaluations of $\left(\log a_{k}\right)^{2}$ and using the functional equation $L(z)+L(1-z)=\pi^{2} / 6$ to tidy up the resulting expression.

In a similar fashion, each of the identities (P1) to (P11) yields the corresponding dilogarithm relation in the list (L1) to (L11). A propos Lewin's conjecture (L13), the coefficient of $q^{k}$ in the power series

$$
\sum_{n=0}^{\infty} \frac{\left(-q^{3} ; q^{3}\right)_{n-1} q^{n}}{(-q)_{n-1}(q)_{2 n}}
$$




$$
2 k^{1 / 2}\left\{\frac{3}{2} L\left(1-\theta^{2}\right)+\frac{1}{3} L\left(1-\theta^{3}\right)-L(1-\theta)-\frac{1}{6} L\left(1-\theta^{6}\right)\right\}^{1 / 2},
$$

with $\theta=2 \cos 4 \pi / 9$. However, the theorem cannot be applied to (P13) because the terms of the series there do not have positive coefficients. This makes it possible for cancellation to occur between the terms, and the identity shows that this does in fact occur to a rather surprising extent.

\section{References}

[1] G. E. Andrews, The theory of partitions, Addison-Wesley, 1976.

[2] W. N. Bailey, On the simplification of some identities of the Rogers-Ramanujan type, Proc. London Math. Soc. (3) 1 (1951), pp. 217-221.

[3] L. Lewin, Polylogarithms and associated functions, Nortli-Holland, 1981.

[1] - The dilogarithm in algebraio fields, J. Austral. Math. Soc. Series A, 33 (1982), pp. $302-330$

[5] B. Richmond and G. Szekeres, Some formulae related to dilogarithms, the zeto function and the Rogers-Ramanujan identities, ibid. 31 (1981), pp. 362-373.

[6] L. J. Slater, Further identities of the Rogers-Ramanujan type, Proc. London Math. Soc. (2) 54 (1951), pp. 147-167.

[7] G. Szekeres, Some asymptotic formulae in the theory of partitions II, Quart. J. Math. (2) 4 (1953), pp. 96-111.

[8] G. N. Watson, A note anz Spence's logarithmic transcendant, Quart. J. Math. Oxford Ser. 8 (1937), pp. 39-42.

SCHOOL OF MATHEMATTCS

UNIVERSITY OF NEW SOUTH WALE

Eensington, New. South Wates, 2033

Australia

\section{A generalization of Hasse's generalization of the Syracuse algorithm}

by

K. R. MatdHews and A. M. Watrs (Brisbane, Australia)

1. Introduction. In 1978, H. Miöller [6] discussed an algorithm due to Hasse: Let $m$ and $d$ be relatively prime positive integers, $d_{d} \geqslant 2 ; R_{d}$ is a complete set of residues mod $d$, not including a representative of the multiples of $d ; N_{d}=\{n \in Z \mid d \nmid n\}$. Then $H: N_{d} \rightarrow N_{d}$ is defined by

$$
H(x)=\frac{m x-r}{d^{\alpha}},
$$

where $m x-r=d^{\alpha} M, \alpha \geqslant 1, a \nmid M, r \in R_{d}$. (It is assumed that $r \in R_{d} \Rightarrow m \nmid r$, to ensure $H$ is well-defined.)

Möller conjectured that the sequence of iterates $\left(H^{k}(n)\right)_{k \geqslant 0}$ is periodic for all $n \in N_{d}$ if and only if $m<d^{d /(d-1)}$ and that the set of pure periods is finite for each ehoice of $m, d$ and $R_{d}$. (See Terras [7], [8], Everett [3], Crandall [2] for the special case $d=2, R_{d}=\{-1\}, m=3$ known as the Syracuse algorithm, and Heppner [4] for the general case.)

Closely related to $H$ is the mapping $T: Z \rightarrow Z$ defined by

(1.2) $T(x)=\left\{\begin{array}{lll}(m x-r) / d & \text { if } d \nmid x, \text { where } m x \equiv r(\bmod d), r \in R_{d}, \\ x / d & \text { if } a \mid x .\end{array}\right.$

In fact $H^{k}(n)=T^{\sigma_{k}}(n)$, where (using Möller's notation)

$$
H^{k}(n)=\frac{m H^{k-1}(n)-r_{k-1}}{d^{\alpha_{k}}} \text { and } \quad \sigma_{k}=\sum_{i=0}^{k} \alpha_{i} .
$$

In the present paper a more symmetric mapping which generalizes $T$ is studied. Let $d, m_{1}, \ldots, m_{d}$ be positive integers, $d \geqslant 2, \operatorname{ged}\left(m_{i}, d\right)=1$ for $i=1, \ldots, d ; R_{d}=\left\{x_{1}, \ldots, x_{d}\right\}$ is a complete set of residues mod $d$; $r_{i} \in R_{d}$ is defined for $i=1, \ldots, d$ by $m_{i} x_{i} \equiv r_{i}(\bmod d)$. Then $T: Z \rightarrow Z$ is given by

$$
T(x)=\frac{m_{i} x-r_{i}}{d} \text { if } \quad x \equiv x_{i}(\bmod d)
$$

